\title{
Distinct Effects of Acute Pretreatment With Lipophilic and Hydrophilic Statins on Myocardial Stunning, Arrhythmias and Lethal Injury in the Rat Heart Subjected to Ischemia/Reperfusion
}

\author{
S. ČARNICKÁ ${ }^{1}$, A. ADAMEOVÁ ${ }^{2}$, M. NEMČEKOVÁ ${ }^{1}$, J. MATEJÍKOVÁ ${ }^{1}$, D. PANCZA ${ }^{1}$, \\ T. RAVINGEROVÁ ${ }^{1}$
}

${ }^{1}$ Institute for Heart Research, Slovak Academy of Sciences, Centre of Excellence NOREG SAS, Bratislava, Slovak Republic, ${ }^{2}$ Department of Pharmacology and Toxicology, Faculty of Pharmacy, Comenius University, Bratislava, Slovak Republic

Received June 7, 2011

Accepted September 1, 2011

\section{Summary}

Although both lipophilic and more hydrophilic statins share the same pathway of the inhibition of HMG-CoA reductase, their pleiotropic cardioprotective effects associated with the ability to cross cellular membranes, including membranes of heart cells, may differ. To test this hypothesis, isolated rat hearts were Langendorff-perfused either with simvastatin $(\mathrm{S}, 10 \mu \mathrm{mol} / \mathrm{l})$ or pravastatin $(P, 30 \mu \mathrm{mol} / \mathrm{l}), 15 \mathrm{~min}$ prior to ischemia. Control untreated hearts $(C)$ were perfused with perfusion medium only. Postischemic contractile dysfunction, reperfusion-induced ventricular arrhythmias and infarct size were investigated after exposure of the hearts to 30-min global ischemia and 2-h reperfusion. Both lipophilic $S$ and hydrophilic $P$ reduced the severity of ventricular arrhythmias (arrhythmia score) from $4.3 \pm 0.2$ in $C$ to $3.0 \pm 0$ and $2.7 \pm 0.2$ in $S$ and $P$, respectively, (both $\mathrm{P}<0.05)$, decreased the duration of ventricular tachycardia and suppressed ventricular fibrillation. Likewise, the extent of lethal injury (infarct size) determined by tetrazolium staining and expressed in percentage of risk area, was significantly lower in both treated groups, moreover, the effect of $P$ was more pronounced $(27 \pm 2 \%$ and $10 \pm 2 \%$ in $S$ and $P$ groups, respectively, vs. $42 \pm 1 \%$ in $C$; $P<0.05$ ). In contrast, only $S$, but not $P$, was able to improve postischemic recovery of left ventricular developed pressure (LVDP; $48 \pm 12 \%$ of preischemic values vs. $25 \pm 4 \%$ in $C$ and $21 \pm 7 \%$ in $P$ groups; $P<0.05$ ). Our results suggest that differences in water solubility of statins indicating a different ability to cross cardiac membranes may underlie their distinct cardioprotective effects on myocardial stunning and lethal injury induced by ischemia/reperfusion.

\section{Key words}

Statins - Water solubility - Pleiotropic effects - Rat heart • Ischemia

\section{Corresponding author}

T. Ravingerová, Institute for Heart Research, Slovak Academy of Sciences, POB 104, Dúbravská cesta 9, 84005 Bratislava, Slovak Republic. Fax: +421 25477 6637. E-mail: usrdravi@savba.sk

Statins have been initially developed to reduce the synthesis of endogenous cholesterol by the inhibition of the 3-hydroxy-3-methylglutaryl coenzyme A reductase (HMG-CoA reductase) in the liver. Now it is generally accepted that they have a wide variety of pleiotropic actions, besides the remarkable hypolipidemic effects, which significantly contribute to the reduction of the incidence of ventricular arrhythmias-associated sudden cardiac death in subjects with and without coronary artery disease (for review see Ramasubbu et al. 2008, Abuissa et al. 2009). These beneficial non-lipid effects of statins are mainly mediated by blocking the synthesis of isoprenoids through a common pathway as cholesterol that results in the inhibition of small GTP-binding proteins (Ras, Rac and Rho) regulating endothelial dysfunction, oxidative stress, inflammation, thrombogenesis and metabolism (McFarlane et al. 2002, Takemoto and Liao 2001). In accordance, experimental studies have clearly shown that statins reduce myocardial electrical disturbances irrespective of their lipid-lowering 
activity and are able to attenuate postischemic cardiac contractile dysfunction in subjects without lipid disorders (Adameová et al. 2009, Ravingerová et al. 2009, Ikeda et al. 2003, Chen et al. 2003).

Statins, a diverse group of natural and synthetic drugs, differ in chemical structure and pharmacokinetic profile, in particular, water solubility, which may determine their lipid-modifying efficacy. In fact, lipophilic statins (simvastatin, atorvastatin, lovastatin) enter hepatocytes non-selectively by passive diffusion, while hydrophilic statins (rosuvastatin and pravastatin) are more hepatoselective due to active carrier-mediated process (Schachter 2005, Hamelin and Turgeon 1998). Data from comparative trials have confirmed that on a miligram basis, rosuvastatin is the most efficient statin for lowering LDL-cholesterol, followed by atorvastatin, simvastatin and pravastatin (Jones et al. 2003). In addition, it is apparent that although both types of statins, lipophilic and hydrophilic, share the same pathway of the inhibition of HMG-CoA reductase, such different pharmacokinetic characteristic may underlie their ability to cross cellular membranes including membranes of cardiomyocytes. To investigate the efficacy of two different types of statins on myocardial stunning, arrhythmias and lethal injury induced by ischemia/reperfusion (I/R) and to explore a potential relationship between the differences in the cardioprotective effects of statins and their chemical structure, in the present study we used two structurally distinct drugs, lipophilic simvastatin (S) and hydrophilic pravastatin $(\mathrm{P})$.

Male Wistar rats (250-300 g body weight), fed a standard diet and tap water ad libitum, were employed. All studies were performed in accordance with the Guide for the Care and Use of Laboratory Animals published by US National Institutes of Health (NIH publication No 8523, revised 1996).

The hearts of anesthetized (sodium pentobarbitone, $60 \mathrm{mg} / \mathrm{kg}$, i.p) animals were rapidly excised and perfused at $37{ }^{\circ} \mathrm{C}$ in the Langendorff mode at a constant perfusion pressure of $73 \mathrm{~mm} \mathrm{Hg}$. The perfusion solution was a modified Krebs-Henseleit buffer gassed with $95 \% \mathrm{O}_{2}$ and $5 \% \mathrm{CO}_{2}$ (pH 7.4) containing (in $\mathrm{mM}$ ): $\mathrm{NaCl} 118.0 ; \mathrm{KCl} 3.2 ; \mathrm{MgSO}_{4} 1.2 ; \mathrm{NaHCO}_{3}$ 25.0; $\mathrm{KH}_{2} \mathrm{PO}_{4} 1.18 ; \mathrm{CaCl}_{2}$ 2.5; glucose 7.0. An epicardial electrogram was registered by means of two electrodes attached to the apex of the heart and the aortic cannula. Left ventricular (LV) pressure was measured by means of a non-elastic water-filled balloon inserted into the $\mathrm{LV}$ cavity and connected to a pressure transducer (MLP844, ADInstruments). LV systolic pressure, diastolic pressure (LVDiP), developed pressure (LVDP; systolic minus diastolic pressure), maximal rates of pressure development and fall, heart rate and coronary flow were measured during stabilization pre-ischemic period and continuously recorded until the end of experiment. Heart function was analyzed using PowerLab/8SP Chart 7 software (ADInstruments).

Global ischemia was induced by clamping of aortic inflow for $30 \mathrm{~min}$ and followed by reperfusion for the evaluation of postischemic recovery of contractile function after 40-min reperfusion. Susceptibility to reperfusion-induced ventricular tachyarrhythmias, such as ventricular tachycardia (VT) and fibrillation (VF), as well as arrhythmia severity (assessed by a 5-point scoring system according to the most severe form of arrhythmia that occurred in each individual heart; Curtis and Walker 1988) was evaluated during 10-min reperfusion. The size of infarction (IS) was determined after 2-h reperfusion by staining with 2,3,5-triphenyltetrazolium chloride and a computerized planimetric method as described earlier (Ravingerová et al. 2009). The IS was expressed as percentage of the LV area.

In drug-treated groups, simvastatin $(10 \mu \mathrm{mol} / \mathrm{l})$ and pravastatin $(30 \mu \mathrm{mol} / \mathrm{l})$ were given as components of the perfusion medium $15 \mathrm{~min}$ prior to ischemia. The rationale for the used dose of statins was based on the evidence that simvastatin is more potent than pravastatin, thereby allowing its administration at the lower dose to achieve the effects on the HMG-CoA reductase that are comparable with the effects of another statin (Schachter 2005). The hearts of the untreated group (C) were perfused for additional 15 min with perfusion medium only.

The data were expressed as means \pm S.E.M. One-way ANOVA and subsequent Student-NewmanKeuls test, as well as Mann-Whitney U test and Fisher's exact test were used where appropriate. Differences were considered as significant at $\mathrm{P}<0.05$.

No significant differences in the values of coronary flow and hemodynamic parameters between the control non-treated and treated groups were observed at baseline and after treatment (data not shown). Acute simvastatin administration prior to ischemia significantly increased postischemic recovery of LVDP $(48 \pm 12 \%$ of preischemic values vs. $25 \pm 4 \%$ in $\mathrm{C}$ groups; $\mathrm{P}<0.05$ ) (Fig. 1A). Likewise, significantly lower postischemic values of LVDiP in the S-treated group (Fig. 1B) 
A

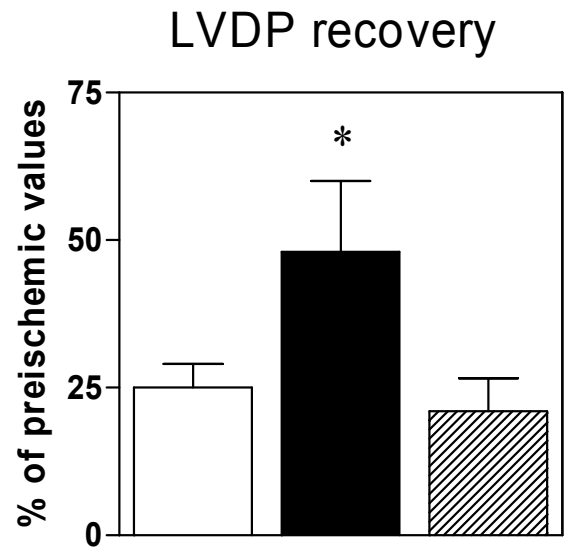

C

Size of infarction

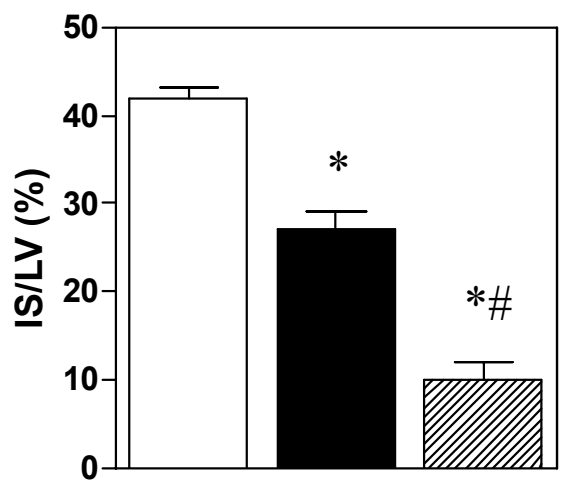

B

LVDiP recovery

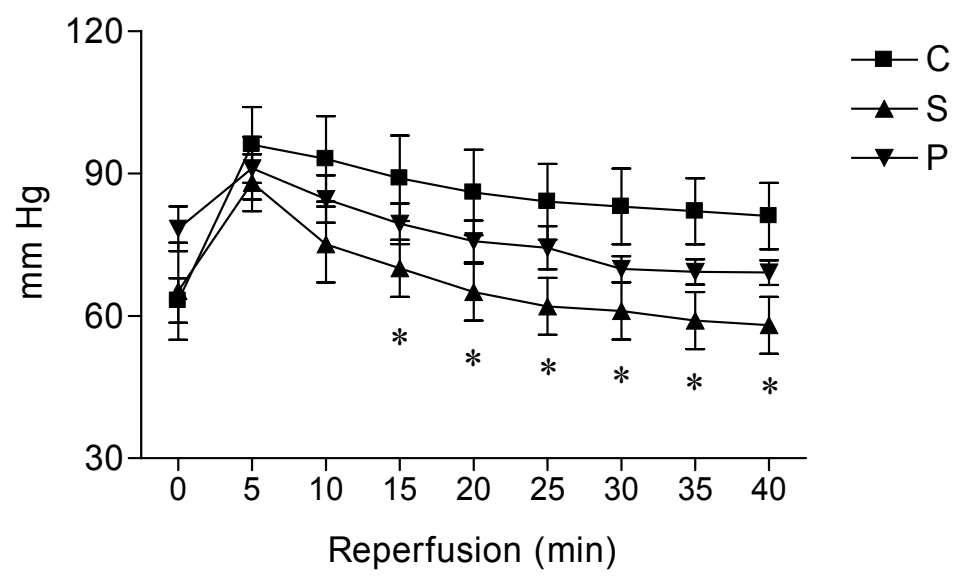

D

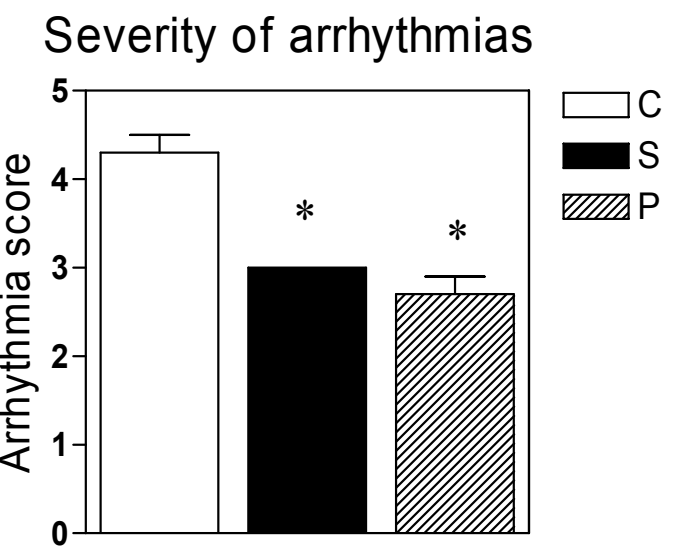

Fig. 1. Effect of acute pretreatment with simvastatin (S) and pravastatin $(P)$ on postischemic recovery of left ventricular developed pressure (LVDP; A), left ventricular diastolic pressure (LVDiP; B), size of infarction (IS) expressed as percentage of left ventricular area (LV; C) and the severity of ventricular arrhythmias (D) in the hearts of normocholesterolemic rats. Empty bars - untreated control hearts, filled bars - S-treated hearts, hatched bars - P-treated hearts. Values are means \pm S.E.M. from $10-12$ hearts per group. * $\mathrm{P}<0.05$ vs. untreated control group; \# $\mathrm{P}<0.05$ vs. simvastatin-pretreated group.

indicated an improvement of the diastolic function and attenuated contracture. However, a reduction of myocardial stunning was not observed in the pravastatintreated hearts; the values of LVDP at $40 \mathrm{~min}$ of reperfusion were comparable with those in the control group ( $21 \pm 7 \%$ in $\mathrm{P}$ vs. $25 \pm 4 \%$ in $\mathrm{C}$ group; $\mathrm{P}>0.05$; Fig. 1A) and diastolic dysfunction was not mitigated (Fig. 1B). On the other hand, postischemic recovery of coronary flow was significantly higher in the $\mathrm{P}$ group than in $\mathrm{C}(80 \pm 9 \%$ and $56 \pm 9 \%$, respectively; $\mathrm{P}<0.05)$, while it was not modified by treatment with simvastatin ( $\mathrm{P}>0.05$ vs. $\mathrm{C}$ ) (Table 1). This effect of pravastatin is in line with the effects of a less hydrophilic fluvastatin that restored function of endothelium within six weeks
(Eichstädt et al. 1995). It could be hypothesized that in our study, specific endothelium-related effects of statins, which are mediated through an increase of the activity/expression of constitutive endothelial nitric oxide synthase (eNOS) and a reduction of caveolin-1, could attenuate decreased NO bioavaibility and thus increase myocardial perfusion (Pelat et al. 2003, Wolfrum et al. 2003, Feron et al. 2001).

The size of myocardial infarction (Fig. 1C) in both treated groups was significantly lower in comparison to the untreated group (IS/LV; $27 \pm 2 \%$ and $10 \pm 2 \%$ in $\mathrm{S}$ and $\mathrm{P}$ groups, respectively, vs. $42 \pm 1 \%$ in $\mathrm{C}$; $\mathrm{P}<0.05$ ). Moreover, the effect of pravastatin on the extent of lethal injury was more pronounced than the effect of 
Table 1. The effects of treatment with simvastatin and pravastatin on postischemic recovery of left ventricular diastolic pressure (LVDiP), heart rate (HR, in \% of preischemic values), coronary flow (CF, in \% of preischemic values) and reperfusion-induced ventricular tachycardia (VT) and fibrillation (VF) in isolated rat hearts subjected to 30-min global ischemia and 40-min reperfusion.

\begin{tabular}{lcccccc}
\hline \multirow{2}{*}{ Group } & \multicolumn{3}{c}{ Recovery } & \multicolumn{2}{c}{ Incidence (\%) } & Duration (s) \\
\cline { 2 - 6 } & $\begin{array}{c}\text { LVDiP } \\
\mathbf{( m m H g )}\end{array}$ & HR (\%) & CF (\%) & VT & VF & VT \\
\hline Control & $81 \pm 7$ & $82 \pm 4.8$ & $56 \pm 9$ & 100 & 83 & $143 \pm 28$ \\
Simvastatin & $58 \pm 6^{*}$ & $90 \pm 3$ & $72 \pm 17$ & 100 & $0^{*}$ & $57 \pm 24^{*}$ \\
Pravastatin & $69 \pm 3$ & $75 \pm 8$ & $80 \pm 9 *$ & 71 & $0^{*}$ & $8 \pm 3^{*} \#$ \\
\hline
\end{tabular}

Data are means from 10-12 hearts per group \pm S.E.M. * $\mathrm{P}<0.05$ vs. control untreated group; $\# \mathrm{P}<0.05$ vs. simvastatin-treated group.

simvastatin $(\mathrm{P}<0.05$; $\mathrm{P}$ vs. $\mathrm{S})$. A smaller infarct size in the treated animals was associated with the reduced severity of ventricular arrhythmias (Fig. 1D, Table 1). Although arrhythmias occurred in both S and P-treated groups, they were less severe in comparison to those in the untreated $\mathrm{C}$ group; the incidence of ventricular fibrillation was completely suppressed, duration of ventricular tachycardia was shorter (Table 1) and arrhythmia severity score (Fig. 1D) was lower in both treated groups ( $3 \pm 0$ and $2.7 \pm 0.2$ in $\mathrm{S}$ and $\mathrm{P}$ groups, respectively, as compared with $4.3 \pm 0.2$ the controls; both $\mathrm{P}<0.05$ ). There were no substantial differences in the antiarrhythmic efficacy between both drugs except shorter duration of VT in the $\mathrm{P}$ group $(\mathrm{P}<0.05$, vs. $\mathrm{S} ;$ Table 1$)$.

In the present study we investigated the role of water solubility, an important pharmacokinetic property of two statins, in pleiotropic cardioprotective effects, such as antiarrhythmic action, infarct size limitation and the ability to restore cardiac contractile function upon I/R. Although both lipophilic simvastatin and hydrophilic pravastatin reduced electrical instability, they differed in the extent of their infarct size-limiting effect. Pravastatin conferred more effective anti-infarct protection that could be partially explained by an improved restoration of myocardial perfusion. On the other hand, only simvastatin but not pravastatin attenuated myocardial contractile dysfunction. Suppression of ventricular arrhythmias upon statin treatment is in accordance with other studies which have suggested that increase of NO bioavailability, antiinflammatory and antioxidative effects as well as regulation of thrombus formation may indirectly underlie antiarrhythmic effects of statins (Takemoto and Liao 2001, McFarlane et al. 2002). In addition to these effects, other statins-mediated antiarrhythmic mechanisms, such as normalization of the electrophysiological disturbances associated with heterogeneity in ventricular repolarization as well as of the heart rate can be also considered (Vrtovec et al. 2005, Mark et al. 2000, Pehlivanidis et al. 2001, Pelat et al. 2003). Recently, we have suggested another mechanism of anti-arrhythmic action of statins; simvastatin given for one week altered the calcium channel composition indicating a reduction of calcium influx into the cardiomyocytes (Szobi et al. 2011). This was shown in both hypercholesterolemic and normocholesterolemic rats confirming the effects of simvastatin independent on lipid-lowering activity. It should be noted that both statins used in the present study were given acutely prior to the induction of ischemia, however, in all above mentioned studies, statin treatment lasted for several days. From the foregoing discussion it appears that octanol-water coefficient indicating the degree of lipophility of statin, is unlikely to determine the efficacy of arrhythmia development. On the other hand, statin lipophility seems to affect the regulation of energy metabolism. In fact, Satoh and Ichihara (2000) demonstrated that cerivastatin, atorvastatin and fluvastatin, but not hydrophilic pravastatin reduced myocardial ATP levels in the reperfused heart of the rats treated for 3 weeks. Of note, these three lipophilic compounds worsened the segment shortening in the reperfused myocardium suggesting that myocardial stunning is associated with the lower ATP content in subjects chronically treated with lipophilic statins. These results are not in line with the findings of the present study; simvastatin but not pravastatin improved postischemic recovery of contractile fucntion. Likewise, only simvastatin considerably reduced postischemic diastolic contracture indicating its greater lusitropic effect. Interestingly, Szárszoi et al. (2008) have shown that administration of simvastatin given during reperfusion is able to restore the recovery of cardiac 
contractility; however, chronic simvastatin treatment failed to exert such cardioprotection. Thus, the duration of statin treatment seems to be an important factor with respect to the restoration of mechanical function of the heart. Although a short-term 7-day treatment with simvastatin, as well as its administration before/during $\mathrm{I} / \mathrm{R}$ attenuates cardiac contractile dysfunction, chronic several-week premedication of rats induces myocardial stunning (Adameová et al. 2009, Szárszoi et al. 2008). In contrast, Kocsis et al. (2008) have reported that lovastatin, a statin with a lower octanol-water coefficient than that of simvastatin, is cardioprotective when given chronically but not acutely. It is thus apparent that water solubility besides the duration of treatment may determine the restoration of heart function upon I/R. In addition, some discrepances in the efficacy of statins in terms of mechanical function of the heart may be also associated with the dose of statin as all authors of the previously mentioned studies used a different dosage regiment. It should be also pointed out that this physicalchemical property, water solubility, of statins is likely to be associated with undesirable effects, such as myalgia, myopathy and rabdomyolysis, which incidence was the highest in the deregistered most lipophilic cerivastatin, a statin known to exert a passive widespread tissue distribution due to the highest octanol-water coefficient (Staffa et al. 2002, Furber and Pitt 2001).

In conclusion, acute treatment with lipophilic simvastatin and hydrophilic pravastatin suppressed the severity of reperfusion-induced tachyarrhythmias and attenuated lethal injury in the rat hearts. While infarct size-limiting effect was more pronounced in pravastatintreated hearts, only simvastatin improved postischemic recovery of myocardial function. Consideration of the physical-chemical and pharmacokinetic properties of statins may provide a basis for a rational choice of a preferable agent to prevent/treat different manifestations of acute myocardial ischemia including arrhythmias, myocardial infarction and contractile dysfunction.

\section{Conflict of Interest}

There is no conflict of interest.

\section{Acknowledgements}

The authors are thankful to Mrs. V. Haššová, I. Blažíčková, and I. Formánková for their excellent technical assistance. This study was supported by grants VEGA SR 1/0620/10, 2/0054/11 and APVV-LPP-0393-09.

\section{References}

ABUISSA H, O'KEEFE JH, BYBEE KA: Statins as anti-arrhythmics: a systematic review part II: effects on risk of ventricular arrhythmias. Clin Cardiol 32: 549-552, 2009.

ADAMEOVÁ A, HARČÁROVÁ A, MATEJÍKOVÁ J, PANCZA D, KUŽELOVÁ M, ČARNICKÁ S, ŠVEC P, BARTEKOVÁ M, STYK J, RAVINGEROVÁ T: Simvastatin alleviates myocardial contractile dysfunction and lethal ischemic injury in rat heart independent of cholesterol-lowering effects. Physiol Res 58: 449-454, 2009.

CHEN J, NAGASAWA Y, ZHU BM, OHMORI M, HARADA K, FUJIMURA A, HASHIMOTO K: Pravastatin prevents arrhythmias induced by coronary artery ischemia/reperfusion in anesthetized normocholesterolemic rats. J Pharmacol Sci 93: 87-94, 2003.

CURTIS MJ, WALKER MJ: Quantification of arrhythmias using scoring systems: an examination of seven scores in an in vivo model of regional myocardial ischaemia. Cardiovasc Res 22: 656-665, 1988.

EICHSTÄDT HW, ESKÖTTER H, HOFFMAN I, AMTHAUER HW, WEIDINGER G: Improvement of myocardial perfusion by short-term fluvastatin therapy in coronary artery disease. Am J Cardiol 76: 122A-125A, 1995.

FERON O, DESSY C, DESAGER JP, BALLIGAND JL: Hydroxy-methylglutaryl-coenzyme A reductase inhibition promotes endothelial nitric oxide synthase activation through a decrease in caveolin abundance. Circulation 103: 113-118, 2001.

FURBERG, CD, PITT, B: Withdrawal of cerivastatin from the world market. Curr Control Trials Cardiovasc Med 2: 205-207, 2001.

HAMELIN BA, TURGEON J: Hydrophilicity/lipophilicity: relevance for the pharmacology and clinical effects of HMG-CoA reductase inhibitors. Trends Pharmacol Sci 19: 26-37, 1998.

IKEDA Y, YOUNG LH, LEFER AM: Rosuvastatin, a new HMG-CoA reductase inhibitor, protects ischemic reperfused myocardium in normocholesterolemic rats. J Cardiovasc Pharmacol 41: 649-656, 2003. 
JONES PH, DAVIDSON MH, STEIN EA, BAYS HE, MCKENNEY JM, MILLER E, CAIN VA, BLASETTO JW; STELLAR STUDY GROUP: Comparison of efficacy and safety of rosuvastatin versus atorvastatin, simvastatin, and pravastatin across doses (STELLAR Trial). Am J Cardiol 92: 152-160, 2003.

KOCSIS GF, PIPIS J, FEKETE V, KOVÁCS-SIMON A, ODENDAAL L, MOLNÁR E, GIRICZ Z, JANÁKY T, VAN ROOYEN J, CSONT T, FERDINANDY P: Lovastatin interferes with the infarct size-limiting effect of ischemic preconditioning and postconditioning in rat hearts. Am J Physiol Heart Circ Physiol 294: H2406H2409, 2008.

MARK L, KATONA A: Effect of fluvastatin on QT dispersion: a new pleiotropic effect? Am J Cardiol 85: 919-920, 2000.

MCFARLANE SI, MUNIYAPPA R, FRANCISCO R, SOWERS JR: Pleiotropic effects of statins: lipid reduction and beyond. J Clin Endocrinol Metab 87: 1451-1458, 2002.

PEHLIVANIDIS AN, ATHYROS VG, DEMITRIADIS DS, PAPAGEORGIOU AA, BOULOUKOS VJ, KONTOPOULOS AG: Heart rate variability after long-term treatment with atorvastatin in hypercholesterolaemic patients with or without coronary artery disease. Atherosclerosis 157: 463-469, 2001.

PELAT M, DESSY C, MASSION P, DESAGER JP, FERON O, BALLIGAND JL: Rosuvastatin decreases caveolin-1 and improves nitric oxide-dependent heart rate and blood pressure variability in apolipoprotein E-/- mice in vivo. Circulation 107: 2480-2486, 2003.

RAMASUBBU K, ESTEP J, WHITE DL, DESWAL A, MANN DL: Experimental and clinical basis for the use of statins in patients with ischemic and nonischemic cardiomyopathy. J Am Coll Cardiol 51: 415-426, 2008.

RAVINGEROVÁ T, ADAMEOVÁ A, KELLY T, ANTONOPOULOU E, PANCZA D, ONDREJČÁKOVÁ M, KHANDELWAL VK, ČARNICKÁ S, LAZOU A: Changes in PPAR gene expression and myocardial tolerance to ischaemia: relevance to pleiotropic effects of statins. Can J Physiol Pharmacol 87: 1028-1036, 2009.

SATOH K, ICHIHARA K: Lipophilic HMG-CoA reductase inhibitors increase myocardial stunning in dogs. J Cardiovasc Pharmacol 35: 256-262, 2000.

SCHACHTER M: Chemical, pharmacokinetic and pharmacodynamic properties of statins: an update. Fundam Clin Pharmacol 19: 117-125, 2005.

STAFFA JA, CHANG J, GREEN L: Cerivastatin and reports of fatal rhabdomyolysis. $N$ Engl J Med 346: 539-540, 2002.

SZÁRSZOI O, MALÝ J, OŠŤÁDAL P, NETUKA I, BEŠÍK J, KOLÁŘ F, OŠŤÁDAL B: Effect of acute and chronic simvastatin treatment on post-ischemic contractile dysfunction in isolated rat heart. Physiol Res 57: 793-796, 2008.

SZOBI A, RAJTÍK T, RAVINGEROVÁ T, ČARNICKÁ S, ŠVEC P, ADAMEOVÁ A: Pleiotropic anti-arrhythmic effect of simvastatin is associated with the alterations in expression of $\mathrm{Ca}^{2+}$-cycling proteins. In: Interactive Conference of Young Scientists, Preveda, Bratislava, 2011.

TAKEMOTO M, LIAO JK: Pleiotropic effects of 3-hydroxy-3-methylglutaryl coenzyme a reductase inhibitors. Arterioscler Thromb Vasc Biol 21: 1712-1719, 2001.

VRTOVEC B, OKRAJSEK R, GOLICNIK A, FERJAN M, STARC V, RADOVANCEVIC B: Atorvastatin therapy increases heart rate variability, decreases QT variability, and shortens QTc interval duration in patients with advanced chronic heart failure. J Card Fail. 11: 684-690, 2005.

WOLFRUM S, GRIMM M, HEIDBREDER M, DENDORFER A, KATUS HA, LIAO JK, RICHARDT G: Acute reduction of myocardial infarct size by a hydroxymethyl glutaryl coenzyme A reductase inhibitor is mediated by endothelial nitric oxide synthase. J Cardiovasc Pharmacol 41: 474-480, 2003. 